\title{
FACTORES DEL AMBIENTE FAMILIAR PREDICTORES DE DEPRESIÓN EN ADOLESCENTES ESCOLARES: ANÁLISIS POR SEXO
}

\author{
Claudia Teresita Ruiz Cárdenas, Juan Jiménez Flores, Mirna García Méndez, \\ Luz María Flores Herrera y Hugo Dayran Trejo Márquez \\ Universidad Nacional Autónoma de México \\ México
}

\begin{abstract}
RESUMEN
Se evaluó la relación entre ambiente familiar y depresión. Participaron 391 adolescentes ( 14 a 16 años de edad) de ambos sexos, estudiantes de escuelas secundarias públicas de la CDMX. Se aplicó la Escala de Ambiente Familiar para Adolescente, formada por cinco factores: conflicto entre los padres, falta de comunicación familiar, falta de reglas y normas, hostilidad y aceptación familiar, con Alpha de .899 y validez de constructo. Para evaluar la depresión se utilizó la escala D del Inventario Multifásico de la Personalidad Minnesota para Adolescentes. La comparación de medias identificó diferencias significativas en la depresión y en los factores conflicto entre los padres, falta de comunicación y hostilidad. El análisis de regresión múltiple indica que las variables predictoras de depresión en mujeres son: falta de reglas y norma, falta de comunicación familiar y aceptación familiar; mientras que en los varones son: falta de aceptación familiar y conflictos entre los padres.
\end{abstract}

Palabras Clave:

depresión, adolescencia, ambiente familiar, comunicación, conflicto entre los padres.

\section{FAMILY ENVIRONMENT FACTORS PREDICTORS OF DEPRESSION IN SCHOOL ADOLESCENTS: ANALYSIS BY SEX}

\begin{abstract}
The study aim known the relationship between the family environment and adolescent's depression. Were 391 adolescents (14 to 16 years old) of both sexes, who studied in middle level schools of Mexico City. The instruments was the Adolescence Family Environment Scale (Ruiz-Cárdenas, Reidl \& Gallegos, 2017), which distributed in five factors: relationship between parents, family communication, rules and standards, hostility, and family acceptance, which account for $52 \%$ of the variance with a global Cronbach's Alpha of .899 and construct validity. The scale D, depression of the Multifactor Inventory of the Minnesota Personality Adolescent used to assess depression. The results obtained show significant differences between men and women. Multiple regression analysis presented as family environment variables that predict depression in women the lack of family communication and family norms, while teenage male variables that predict depression are lack of family acceptance and conflictive between the parents.
\end{abstract}

Keywords:

depression, adolescence, family environment, communication, parental conflict

Bitácora del ARtículo:

| Recibido: 15 de Noviembre de 2018 | Aceptado: 6 de Septiembre de 2019 | Publicado en línea: Enero-Junio de 2020 | 


\title{
Autoría y Derechos de Propiedad Intelectual
}

\author{
FACTORES DEL AMBIENTE FAMILIAR PREDICTORES DE DEPRESIÓN EN \\ ADOLESCENTES ESCOLARES: ANÁLISIS POR SEXO
}
Claudia Teresita Ruiz Cárdenas, Juan Jiménez Flores, Mirna García Méndez, Luz María Flores Herrera y Hugo Dayran Trejo Márquez Universidad Nacional Autónoma de México México

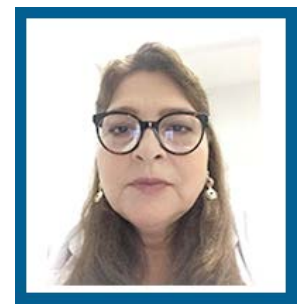

Claudia Teresita Ruíz Cárdenas FES- Zaragoza, UNAM Correo: claudiatruiz@hotmail.com

Doctorado en Clínica Psicoanalítica. Investigador en el Instituta Nacional de Salud Pública; Profesor investigador de Tiempo Completo en la FES Zaragoza; Psicólogo clínico especializado en el Centro Comunitario de Salud Mental Cuauhtémoc, Servicios de Atención Psiquiátrica (SAP).

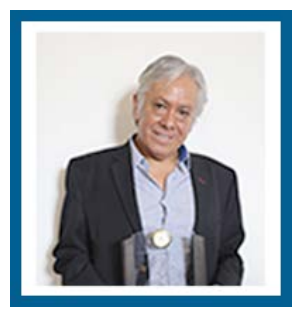

Juan Jiménez Flores FES- Zaragoza, UNAM Correo: jimenezj19@hotmail.com

Profesor en la Carrera de Psicología en la FES Zaragoza. Docente en el Posgrado del Instituto Salesiano de Estudios Superiores. Coordinador del Programa de Prevención Psicológica de la FES Zaragoza, UNAM. Perito en psicología forense en la Agencia de Servicios Periciales Especializados.

\section{CONTRIBUCIÓN DE LOS AutORES}

Claudia Teresita Ruiz Cárdenas: Revisión bibliográfica, aplicación de instrumentos, análisis estadísticos e interpretación de resultados. | uan Jiménez Flores: desarrollo de metodología. | Mirna García Méndez: discusión de resultados. | Luz María Flores Herrera: revisión bibliográfica y presentación de resultados. | Mtro. Hugo Dayran Trejo Márquez: redacción y corrección de estilo.

\section{AGRADECIMIENTOS}

El presente estudio agradece el apoyo brindado de la Carrera de Psicología de la FES Zaragoza y al Centro Comunitario de Salud Mental Cuauhtémoc de los Servicios de Atención Psiquiátrica (SAP).

\section{DATOS DE FILIACIÓN DE LOS AUtORES}

Claudia Teresita Ruiz Cárdenas está adscrita a la Facultad de Estudios Superiores Zaragoza, UNAM y al Centro Comunitario de Salud Mental Cuauhtémoc. | Juan Jiménez Flores, Mirna García Méndez y Luz María Flores Herrera están afiliados a la la Facultad de Estudios Superiores Zaragoza | Hugo Dayran Trejo Márquez está afiliado al Centro Comunitario de Salud Mental Cuauhtémoc.

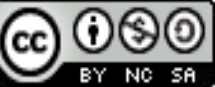

Copyright: (c) 2020 Ruíz-Cárdenas, C.T., Jiménez-Flores, J., García-Méndez, M. \& Flores-Herrera H.D.

Este es un artículo de acceso abierto distribuido bajo los términos de la licencia Creative Commons Reconocimiento-NoComercial 4.0 Internacional, por lo que su contenido gráfico y escrito se puede compartir, copiar y redistribuir total o parcialmente sin necesidad de permiso expreso de sus autoras con la única condición de que no se puede usar con fines directamente comerciales y los términos legales de cualquier trabajo derivado deben ser los mismos que se expresan en la presente declaración. La única condición es que se cite la fuente con referencia a la Revista Digital Internacional de Psicología y Ciencia Social y a sus autoras. 


\section{TABLA DE CONTENIDO}

MÉTODO

Objetivos, 103

Participantes, 103

Variables, 104

Hipótesis, 104

Instrumentos, 104

Diseño, 104

Procedimiento, 104

Resultados

105

Discusión

106

REFERENCIAS 


\section{L}

a depresión es uno de los trastornos mentales más frecuentes; afecta a millones de personas en el (Organización Mundial de la Salud, 2016).

Los informes de la Organización Panamericana de la Salud (2009) para América Latina y el Caribe informan de una prevalencia de $20 \%$ en los trastornos mentales de la población juvenil.

La depresión incrementa la probabilidad de ocurrencia de otros problemas psiquiátricos internalizados y externalizados (González-Forteza, Jiménez-Tapia, Ramos-Lira y Wagner, 2008) o conductas problemáticas, como el trastorno disocial, déficit de atención, ansiedad, trastornos de conducta alimentaria e intento de suicidio (Bella, 2010; Cárdenas, Feria, Vázquez, Palacios y De la Peña, 2010); a esto se agregan el consumo de sustancias como el tabaco y el alcohol (Bella, 2010; Cárdenas et al., 2010; González-González, Juárez, Solís, González-Forteza, Jiménez, Medina-Mora y Fernández-Varela, 2012; Schleicher, Harris, Catley y Nazir, 2009;) con una importante morbilidad que contribuye al deterioro de la calidad de vida de los menores (Moreno y Medina-Mora, 2008). Asimismo se observa una tendencia a incrementar el riesgo para el desarrollo de otros trastornos psiquiátricos en la adultez.

La depresión grave puede llevar al suicidio; a nivel mundial cada año se suicidan más de 800,000 personas; el suicidio es la segunda causa de muerte en el grupo etario de 15 a 29 años (OMS, 2016). Entre 1990 y a la fecha, en México se han incrementado los casos de suicidio (5\% en personas de 5 a 14 años de edad y $74 \%$ entre las de 15 y 24 años) (Benjet, Borges, Medina-Mora, Fleiz-Bautista y Zambrano-Ruiz, 2004). En Ciudad de México, $11.5 \%$ de los jóvenes de 12 a 17 años de edad ha tenido ideación suicida (Borges, Benjet, Medina-Mora, Orozco y Nock, 2008).

Cuando la depresión inicia manera temprana tiende hacia la cronicidad y el deterioro psicosocial. En México la prevalencia de la depresión con inicio temprano tiene una comorbilidad con mayor probabilidad de presentar agorafobia, fobia social, trastorno negativista desafiante, trastorno disocial y dependencia a drogas. Asimismo, se atribuye a la depresión $10 \%$ de la carga global de las enfermedades, estimando que aumentará hasta $15 \%$ en el 2020 (Bridge, Goldstein y Brent, 2006).
La depresión, además de tener mayor prevalencia hoy, cada vez se presenta en población más joven, dando lugar a la necesidad de estudiar el curso de los síntomas depresivos desde la infancia o la adolescencia temprana (Bridge et al., 2006).

Entre las implicaciones que tiene la depresión a lo largo de la vida, están la influencia en el estado de ánimo, el estilo de afrontamiento y la trascendencia de las decisiones y algunos acontecimientos hacia la vida futura, como la continuidad académica, el inicio de la vida profesional o laboral y la vida en pareja y en familia (Bridge et al., 2006; Informe sobre la Salud Mental de Niños y Adolescentes, 2008; Organización Panamericana de la Salud, 2009), por lo que se convierte en un factor de vulnerabilidad para el proyecto de vida de los adolescentes (González-Forteza et al., 2008).

Las diferencias por sexo en la prevalencia de la depresión son importantes; la probabilidad para presentar depresión clínica es dos o tres veces mayor en las mujeres en comparación con los hombres (Benjet, Borges, Medina-Mora, Méndez, Fleiz, Rojas y Cruz, 2009). El análisis de la depresión en adolescentes tiene aspectos interesantes, como las diferencias y similitudes entre hombres y mujeres (Marmorstein y lacono, 2003). Hay datos que indican diferencias en la prevalencia por sexo en la incidencia del trastorno depresivo mayor en la adolescencia con nivel de 2.1 de predominio femenino (Álvarez, Atienza, Heras, Fernández, Ferrer y Goicoechea, 2009).

Los datos han sido consistentes; los trastornos afectivos en general, y en particular la depresión mayor, muestra entre los adolescentes diferencias significativas por sexo (Benjet et al., 2009; Veytia, González, Andrade y Oudhof, 2012). Al respecto se ha indicado la inequidad de género y la cultura como factores asociados con los síntomas depresivos, entre los cuales están diferencias económicas, el cuidado de los hijos, el hogar y la prevalencia de relaciones asimétricas que favorecen la subordinación de la mujer (Hopcroff y Bradley, 2007), así como la femineidad como una variante de la indefensión aprendida, menor estatus social y experimentar menor control en muchas áreas de su vida (Zarragoitía, 2013).

Las manifestaciones clínicas de la depresión varían según la edad de los grupos. La etapa de la adolescencia en que la depresión se observa con mayor frecuencia oscila entre 13 a 15 años de edad, nivel que coincide con las épocas de más baja autoestima de este periodo del crecimiento (Pintado-Machado, Jiménez, Padilla, Guerra y Antelo, 2010).

Los episodios depresivos pueden clasificarse como leves, moderados o graves. En la depresión grave, en el caso del trastorno de depresión mayor (TDM), en las últi- 
mas dos décadas se ha reportado una prevalencia en niños preescolares de $2 \%$, en escolares se calcula entre 8 y $10 \%$, y en adolescentes asciende a 13\% (Garrison, Walter, Cuffe, MacKeown, Anddy y Jackson, 1997). El diagnóstico y el tratamiento del TDM en una edad joven puede reducir su duración y la severidad de los efectos en el funcionamiento adulto (Essau, Conradt y Petermann, 2000).

Entre los factores de riesgo para presentar depresión se encuentran el rendimiento escolar, la dinámica familiar, falta de comunicación entre padres e hijos, carencias afectivas y medidas de control utilizados en el hogar (Galicia, Sánchez y Robles, 2009); también la falta de comunicación en la población adolescente ha sido reportada con mayor peso predictivo en la depresión, reportando cuanto mayor es la comunicación y autonomía percibido por el adolescente tanto del padre como de la madre, menor sintomatología depresiva (Andrade, Betancourt, Vallejo, Segura y Rojas 2012). A esto se suman los factores de riesgo socioculturales, la depresión parental, el conflicto o divorcio mal estructurado, problemas familiares y el permanecer mucho tiempo solos (Rodríguez y Granada, 2007).

En unn estudio respecto a relaciones parentales y depresión, en el cual los autores aplicaron la escala de depresión del Centro de Estudios Epidemiológicos (CES-D), los datos indican que el grupo de adolescentes que puntuaron por arriba del diagnóstico clínico de depresión tuvieron problemas de relaciones con sus padres, inferior intimidad verbal con sus padres, menos cariño físico con sus padres y mayor cantidad de padres con depresión (Field, Diego y Sanders, 2002).

En México, al estudiar los sucesos negativos del área familiar y comparar entre el grupo clínico con depresión y el grupo de adolescentes sin depresión evaluados con la escala clínica 2D de depresión y la escala de contenido Dep A depresión-adolescentes del MMPI-A, se encontró que los adolescentes con depresión tuvieron mayor impacto de sucesos estresantes negativos relacionados con la dinámica familiar, las relaciones sociales, dificultades personales y problemas de conducta, en comparación con el grupo sin depresión (Heredia- Ancona, Lucio-Gómez y Suárez, 2011). La depresión se relaciona de manera estrecha con los conflictos familiares (Álvarez, Ramírez, Silva, Cuffin y Jiménez, 2009).

Las relaciones interpersonales acontecen en un proceso en el que interactúan diversas variables entre el conflicto, el enfrentamiento y la depresión (García-Méndez, Rivera-Aragón, Reyes-Lagunes y Díaz-Loving, 2011). Así, las interacciones conflictivas tienen una asociación importante con la depresión, como la muerte temprana o la separación de los padres (Álvarez et al., 2009; Rosa-Alcázar y Parada-Navas, 2014).

La no aceptación de los padres y las críticas familiares se relacionaban con depresión y problemas de conducta en los adolescentes, siendo la no aceptación de los padres la de mayor importancia predictiva (Rosa-Alcázar y Parada-Navas, 2014). Las situaciones negativas que se viven en la familia, como las interacciones conflictivas, también tienen una asociación importante con la depresión (Veytia et al., 2012).

Se afirma que la presencia frecuente de conflictos entre los miembros de la familia puede influir para desarrollar relaciones satisfactorias en el ambiente familiar y escolar, y se que produzcan bajas expectativas de autoeficacia de tipo académico y social (Galicia, Sánchez y Robles, 2013).

En consecuencia, el presente estudio tiene como objetivo identificar el nivel predictivo para la depresión de las variables del ambiente familiar en los adolescentes e identificar diferencias de acuerdo con el sexo. Es decir, ¿cuál es el nivel predictivo del conflicto entre los padres, el rechazo familiar, la falta de normas y reglas de funcionamiento familiar, la falta de comunicación entre los miembros de la familia y la hostilidad en la depresión de los hombres y mujeres adolescentes?

\section{MÉTodo}

\section{Objetivos}

1) Evaluar si ocurren diferencias en los síntomas depresivos de acuerdo con el sexo.

2) Identificar si hay diferencias en los factores del ambiente familiar en los adolescentes de acuerdo con el sexo.

3) Determinar si los factores del ambiente familiar influyen en la sintomatología depresiva.

\section{Participantes}

La muestra estuvo integrada por 391 adolescentes: 191 hombres $(49 \%)$ y 200 mujeres (51\%), con edades de 14 a 16 años $(M=14.5, D E=0.561 ; 47 \%$ de 14 años, $50 \%$ de 15 años y $3 \%$ de 16 años), todos alumnos de segundo y tercer grado de escuelas secundarias públicas de la zona centro y oriente de Ciudad de México (tabla 1). La selección de los participantes se hizo por muestro aleatorio incidental (Cohen y Swerdlik, 2006); los instrumentos se aplicaron con los adolescentes que acudieron con ese momento. 
Tabla 1.

Variables demográficas.

\begin{tabular}{|cclll|}
\multicolumn{2}{c}{ EDAD } & \multicolumn{3}{c}{ Sexo } \\
\hline 14 & $47 \%$ & Hombres & 191 & $48.8 \%$ \\
\hline 15 & $50 \%$ & Mujeres & 200 & $51.2 \%$ \\
\hline 16 & $3 \%$ & & & \\
\hline
\end{tabular}

\section{Variables}

\section{Variable dependiente: Depresión}

- Medida del índice de insatisfacción general de la propia vida, con sentimientos de desánimo, desesperanza y moral baja. Sus comportamientos incluyen abatimiento y apatía, hipersensibilidad, quejas y problemas físicos (Lucio, Ampudia y Durán, 2004).

\section{Variables independientes:}

- Conflicto entre los padres. Consiste en los patrones de relación que el adolescente percibe entre los padres, en la identificación de problemas, el discurso reiterado de los conflictos, la falta de acuerdos y la falta de afecto que percibe en el entorno familiar (Ruiz-Cárdenas, Reidl y Cazares, 2017).

- Comunicación familiar. Se caracteriza por el tipo de comunicación que el adolescente establece con la familia, en relación con sus actividades cotidianas, emociones y afectos (Ruiz-Cárdenas et al., 2017).

- Falta de reglas y normas. Se refiere a los patrones de relación caracterizados por la falta de límites y respeto en la relación con los miembros de la familia (Ruiz-Cárdenas et al., 2017).

- Hostilidad. Consiste en el comportamiento y actitud intolerante y demandante que el adolescente presenta y observa en el entorno familiar (Ruiz-Cárdenas et al., 2017).

- Aceptación familiar. Es la aceptación, cooperación, presentación de alternativas a los problemas y en el respeto a la individualidad de los miembros de la familia (Ruiz-Cárdenas et al., 2017).

\section{Hipótesis}

1) Estadísticamente hay diferencias en los síntomas de depresión en los hombres y mujeres adolescentes.

2. Hay diferencias estadísticamente significativas en los factores del ambiente familiar de acuerdo con sexo.

3) Los factores del ambiente familiar: conflicto entre los padres, falta de comunicación, falta de reglas y normas, hostilidad y la no aceptación familiar predicen la depresión en adolescentes. Conforme aumenta el valor de los factores del ambiente familiar se incrementa la sintomatología depresiva.

\section{Instrumentos}

La escala de ambiente familiar del adolescente (EAFA) (Ruiz-Cárdenas et al., 2017) es un instrumento diseñado y validado para población mexicana con el objetivo de evaluar la percepción que los adolescentes tienen de su ambiente familiar, formado por 25 reactivos en formato tipo Likert con cuatro opciones de respuesta y distribuidos en cinco factores que explican $52 \%$ de la varianza con un Alpha de Cronbach global de 0.90 y validez de constructo. Los factores obtenidos son: conflicto entre los padres (seis reactivos), falta de comunicación familiar (cinco reactivos); ausencia de normas y reglas (seis reactivos); hostilidad (cinco reactivos) y aceptación familiar (tres reactivos).

El inventario multifásico de la personalidad Minnesota para adolescentes (MMPI-A) (Lucio et al., 2004) fue validado para población mexicana y está integrado por 478 reactivos dicotómicos; fue desarrollado a partir del Minnesota Multiphasic Personality Inventory-Adolescent (Butcher, Williams, Graham, Archer, Tellegen, Ben-Porath y Kaermmer, 1992). Es un instrumento psicológico utilizado en el campo clínico; evalúa los aspectos psicopatológicos del adolescente. Para el presente estudio se consideró la escala básica 2D de depresión, formada por 57 reactivos dicotómicos (cierto o falso); tiene validez de criterio y alfa de Cronbach de 0.76. El puntaje bruto obtenido se transformó en puntaje total, mismo que identificó los niveles "por debajo de lo normal", "normal" y "por arriba de lo normal". Sólo se consideraron los resultados de los participantes que obtuvieron puntajes que permitieran validar sus respuestas a partir de los criterios establecidos en las escalas de validez del instrumento.

\section{Diseño}

Es un estudio de campo, no experimental, de tipo transeccional.

\section{Procedimiento}

La aplicación de los instrumentos fue voluntaria; se hizo de manera colectiva en las aulas, con personal de apoyo previamente capacitado, con la presencia del docente y el personal responsable de la investigación. Se hizo hincapié en que los resultados obtenidos eran anónimos, confidenciales y tendrían un uso sólo estadístico.

Para desarrollar la aplicación de la EAFA y del MMPI-A se elaboraron los oficios correspondientes para 
solicitar la autorización del personal directivo de las escuelas y de los padres mediante el consentimiento informado por escrito. Los datos obtenidos se procesaron para su análisis estadístico, el cual se efectuó en el paquete estadístico SSPS 21.

\section{Resultados}

En el análisis de las diferencias de la depresión de acuerdo con el sexo de los participantes, la comparación de medias obtenida en la escala de depresión presenta diferencias significativas entre los adolescentes hombres y mujeres (tabla 2).

Tabla 2.

Comparación de medias en los rasgos de depresión en hombres y mujeres adolescentes.

\begin{tabular}{|c|c|c|c|c|c|c|}
\hline \multicolumn{3}{|c|}{ HOMBRES } & \multicolumn{2}{|c|}{ Mujeres } & \multirow[b]{2}{*}{$t$} & \multirow[b]{2}{*}{$\mathrm{P}$} \\
\hline Variables & $M$ & $\mathrm{DE}$ & $M$ & $\mathrm{DE}$ & & \\
\hline Depresión & 20.10 & 5.41 & 22.79 & 5.86 & -4.69 & $0.000^{* *}$ \\
\hline${ }^{* *} p<0.01$ & & & & & & \\
\hline
\end{tabular}

Para determinar si hay diferencias estadísticamente significativas por sexo, entre los niveles de normalidad en la escala de depresión se procedió a transformar los valores intervalares a valores categóricos, y de esta manera se hizo el análisis de chi-cuadrado. Los resultados indican que $13 \%$ de las mujeres presenta un puntaje elevado en el rango por arriba de la norma, en comparación a 4.2\% de los varones en la escala de depresión; también se observa en los hombres $62 \%$ por debajo de lo normal (tabla 3 ). integran el ambiente familiar identificando diferencias significativas en tres de los cinco factores: 1) conflicto entre los padres; 2) falta de comunicación, y 3) hostilidad (tabla 4).

Tabla 4

Comparación de medias de los puntajes de ambiente familiar en hombres y mujeres adolescentes.

\begin{tabular}{|c|c|c|c|c|c|c|}
\hline \multicolumn{3}{|c|}{ HOMBRES } & \multicolumn{2}{|c|}{ Mujeres } & \multirow[b]{2}{*}{$\mathrm{t}$} & \multirow[b]{2}{*}{$P$} \\
\hline Variables & $M$ & DE & M & DE & & \\
\hline $\begin{array}{l}\text { Conflicto } \\
\text { entre los } \\
\text { padres }\end{array}$ & 8.92 & 3.47 & 10.28 & 4.19 & -3.38 & $0.001^{* *}$ \\
\hline $\begin{array}{l}\text { Falta de } \\
\text { comunicación }\end{array}$ & 9.68 & 2.17 & 10.33 & 2.78 & -2.59 & $0.010^{* *}$ \\
\hline $\begin{array}{l}\text { Falta de } \\
\text { normas y } \\
\text { reglas }\end{array}$ & 10.21 & 3.54 & 10.15 & 3.51 & 0.148 & 0.882 \\
\hline Hostilidad & 8.74 & 2.75 & 9.39 & 3.11 & -2.17 & $0.030^{*}$ \\
\hline $\begin{array}{l}\text { Aceptación } \\
\text { familiar }\end{array}$ & 9.88 & 2.10 & 9.84 & 2.28 & 0.198 & 0.843 \\
\hline \multicolumn{7}{|c|}{$\mathrm{p}<0.01^{* *} \mathrm{p}<0.05^{*}$} \\
\hline
\end{tabular}
ambiente familiar respecto a los síntomas de depresión de los adolescentes se hizo el análisis de regresión múltiple paso a paso. Las variables independientes integrados en el análisis fueron: 1) conflicto entre los padres; 2) falta de comunicación familiar; 3) falta de normas y reglas; 4) hostilidad, y 5) aceptación familiar, mientras que la variable dependiente fue la depresión. A continuación se muestran los coeficientes de regresión no estandarizados (B), los estandarizados ( $\beta$ ) y los coeficientes de

Tabla 3.

Análisis de chi-cuadrado - Escala de depresión.

\begin{tabular}{|c|c|c|c|c|c|c|c|c|c|}
\hline & \multicolumn{6}{|c|}{ Depresión, 2D } & \multirow{3}{*}{$\mathbf{N}$} & \multirow{3}{*}{$\%$} & \multirow{3}{*}{$x^{2}$} \\
\hline & \multicolumn{2}{|c|}{$\begin{array}{c}\text { Debajo de lo } \\
\text { Normal }\end{array}$} & \multicolumn{2}{|c|}{ NORMAL } & \multicolumn{2}{|c|}{$\begin{array}{c}\text { ARRIBA DE LO } \\
\text { NORMAL }\end{array}$} & & & \\
\hline & $\mathbf{N}$ & $\%$ & $\mathbf{N}$ & $\%$ & N & $\%$ & & & \\
\hline Masculino & 119 & 62.3 & 64 & 33.5 & 8 & 4.2 & 191 & 48.9 & \\
\hline Femenino & 94 & 47 & 80 & 40 & 26 & 13 & 200 & 51.1 & $14.042^{* *}$ \\
\hline $\begin{array}{l}\text { Total } \\
{ }^{* *} \mathrm{p}<0.01\end{array}$ & 213 & 54.5 & 144 & 37 & 34 & 8.7 & 391 & 100 & \\
\hline
\end{tabular}

Con la finalidad de identificar diferencias de acuerdo con el sexo en los factores del ambiente familiar, primero se hizo el análisis de medias de los factores que determinación $\left(R^{\wedge} 2\right)$.

En el primer paso del análisis se incorporó como predictor en la ecuación el conflicto entre los padres; 
explicó $12 \%$ de la varianza $(F 1,357)=50.259, p \leq 05)$; en el segundo paso se incluyó como predictor en la ecuación la aceptación familiar, incrementando la $R^{\wedge} 2$ $=0.161 \mathrm{~F}$ incrementada $(\mathrm{F} \mathrm{1,357)}=34.011, \mathrm{p} \leq 05)$, y en el tercer paso se incluyó como predictor en la ecuación la falta de comunicación, incrementó la $\mathrm{R}^{\wedge} 2=0.177$ $\mathrm{F}$ incrementada $(1,357)=25.30, \mathrm{p} \leq 0.05)($ tabla 5). Es decir, se encontraron relaciones estadísticamente significativas con tres dimensiones del ambiente familiar (conflicto entre padres, aceptación familiar y falta de comunicación) que se relacionaron de modo positivo con la depresión en los adolescentes.

\section{Tabla 5.}

Análisis de regresión múltiple en variables que predicen la depresión (D) en hombres y mujeres.

\section{$\begin{array}{llll}\text { VARIABLE } & B & \text { EE }\end{array}$}

\begin{tabular}{|c|c|c|c|}
\hline $\begin{array}{l}\text { Paso } 1 \\
\text { Conflicto entre los } \\
\text { padres }\end{array}$ & 0.305 & 0.087 & $0.204^{* *}$ \\
\hline $\begin{array}{l}\text { Pa } 14.042^{* *} \text { so } 2 \\
\text { Aceptación familiar }\end{array}$ & -0.501 & 0.143 & $-0.188^{* *}$ \\
\hline $\begin{array}{l}\text { Paso } 3 \\
\text { Falta de comunicación }\end{array}$ & 0.333 & 0.128 & $0.145^{* *}$ \\
\hline \multicolumn{4}{|c|}{$\begin{array}{l}\mathrm{R}^{\wedge} 2=0.124 \text { para el paso } 1(\mathrm{p}<0.01) ; \mathrm{R}^{\wedge} 2=0.161 \text { para el } \\
\text { paso } 2(\mathrm{p}<0.01) ; \mathrm{R}^{\wedge} 2=0.177 \text { para el paso } 3(\mathrm{p}<.01) \\
{ }^{*} \mathrm{p}<0.01\end{array}$} \\
\hline
\end{tabular}

Con el objetivo de identificar diferencias en las variables del ambiente familiar de acuerdo con el sexo, se procedió a desarrollar dos análisis de regresión, uno para los hombres y otro para las mujeres. Para el primer caso el análisis de regresión múltiple consideró como variables independientes a los cinco factores de ambiente familiar y como variable dependiente la depresión. Como primer paso el análisis de regresión múltiple paso a paso en hombres incorporó como predictor en la ecuación la aceptación familiar; explicó $6 \%$ de la varianza $[F(1,176)=11.171, p=.00+5]$. En el segundo paso se incluyó como predictor en la ecuación al conflicto entre los padres; incrementó la $\mathrm{R}^{\wedge} 2=0.088 \mathrm{~F}$ incrementada $[F(1,176)=8.438, p=0.00]$ (tabla 6).

Tabla 6.

Análisis de regresión múltiple en variables que predicen la depresión (D) en hombres.

\begin{tabular}{|c|c|c|c|}
\hline VARIABLE & B & EE & B \\
\hline $\begin{array}{l}\text { Paso } 1 \\
\text { Aceptación familiar }\end{array}$ & -0.507 & 0.191 & $-0.199 * *$ \\
\hline \multicolumn{4}{|c|}{$\begin{array}{l}R^{\wedge} 2=0.060 \text { para el paso } 1(p<0.01) ; R^{\wedge} 2=0.088 \text { para el } \\
\text { paso } 2(p<0.01) ; R^{\wedge} 2=0.088 . \\
{ }^{* *} p<0.01\end{array}$} \\
\hline
\end{tabular}

\section{VARIABLE}

B

EE

B

Paso 2

Conflicto entre los $0.274 \quad 0.118 \quad 0.175^{* *}$ padres

$\mathrm{R}^{\wedge} 2=0.060$ para el paso $1(\mathrm{p}<0.01) ; \mathrm{R}^{\wedge} 2=0.088$ para el paso $2(p<0.01) ; R^{\wedge} 2=0.088$.

${ }^{* *} p<0.01$.

El análisis de regresión múltiple paso a paso en mujeres, en el primer paso se incorporó como predictor en la ecuación la falta de normas y reglas familiares; explicó $17 \%$ de la varianza $[F(1,180)=38.413, p=$ 0.00]; en el segundo paso se incluyó como predictor a la ecuación la falta de comunicación; incrementó la $\mathrm{R}^{\wedge} 2$ $=0.225 \mathrm{~F}$ incrementada $[\mathrm{F}(1,180)=25.810, \mathrm{p}=0.00$ $0]$, y en el tercer paso se incluyó como predictor en la ecuación, con valores negativos, la aceptación familiar; incrementó la $R^{\wedge} 2=0.251 \mathrm{~F}$ incrementada $[F(1,180)=$ 19.760, $p=0.000$ ] (tabla 7).

\section{Tabla 7.}

Análisis de regresión múltiple en variables que predicen la depresión $(D)$ en mujeres.

\begin{tabular}{|c|c|c|c|}
\hline VARIABLE & B & EE & B \\
\hline $\begin{array}{l}\text { Paso } 1 \\
\text { Falta de normas y } \\
\text { reglas }\end{array}$ & 0.315 & 0.145 & $0.187^{* *}$ \\
\hline $\begin{array}{l}\text { Paso } 2 \\
\text { Falta de } \\
\text { comunicación }\end{array}$ & 0.468 & 0.167 & $0.223^{* *}$ \\
\hline $\begin{array}{l}\text { Paso } 3 \\
\text { Aceptación familiar }\end{array}$ & -0.518 & 0.208 & $-0.198^{* *}$ \\
\hline \multicolumn{4}{|c|}{$\begin{array}{l}R^{\wedge} 2=0.177 \text { para el paso } 1(p<0.01) ; R^{\wedge} 2=0.225 \text { para el } \\
\text { paso } 2(p<0.01) ; R^{\wedge} 2=0.251 \text { para el paso } 3(p<0.01) \\
{ }^{* *} p<0.01\end{array}$} \\
\hline
\end{tabular}

Los resultados de los análisis por sexo indicaron que la aceptación familiar y el conflicto entre los padres se relacionan de manera significativa con la depresión en los hombres, mientras que en las mujeres las relaciones fueron con ausencia de normas y reglas, falta de comunicación y aceptación familiar.

\section{DisCusión}

Respecto a los resultados obtenidos se identificó que las mujeres adolescentes presentan tres veces más sintomatología clínica de depresión en comparación con los varones, lo cual es consistente con lo reportado en estudios previos (Benjet et al., 2009; Veytia et al., 2012), con mayor 
vulnerabilidad de las mujeres. Esto se, puede explicar a partir de la teoría de intensificación del rol de género y por la exposición diferencial al estrés (Benjet et al., 2009).

Destaca que gran parte de los hombres adolescentes se ubica debajo de lo normal en la escala de depresión, y sólo una tercera parte de ellos se ubica en el nivel de normalidad, lo que pudiera sugerir una escasa capacidad para ser sensibles a los acontecimientos adversos familiares y las dificultades para reconocer los conflictos familiares y la hostilidad. Esta última creada por lo general por el adolescente y sin la conciencia en el ejercicio de la violencia a partir de la descalificación y la actitud demandante e intolerante de los jóvenes en el entorno familiar, lo que podría constituirse como irritabilidad.

Al analizar los factores que integran el ambiente familiar de acuerdo con el sexo se identificaron diferencias entre los factores "conflicto entre los padres", "falta de comunicación familiar" y "hostilidad", en los que, en comparación con las mujeres, los varones tienen una percepción de menor frecuencia de ellos. La falta de normas y reglas, así como la aceptación familiar, son factores que no muestran diferencias significativas por sexo. Sin embargo, como lo muestra el análisis de regresión múltiple, la falta de normas y reglas es la variable de mayor importancia predictiva para la depresión en las mujeres.

Entre las variables que predicen la depresión, tanto en hombres como en mujeres, se encontró la falta de comunicación, los conflictos entre los padres y falta de aceptación familiar, esto es, los jóvenes que perciben un deficiente ambiente familiar, con falta de comunicación en sus actividades cotidianas, la ausencia de disposición para compartir sus estados emocionales con los miembros de la familia, así como la carencia de vínculos emocionales y sentimientos de cercanía entre la familia, además de patrones de relación que el adolescente percibe de los padres en la identificación de problemas, el discurso reiterado de los conflictos, la falta de acuerdos y la falta de afecto que percibe en el entorno familiar, la falta de aceptación, cooperación, falta de alternativas a la solución de los problemas familiares y la falta de respeto a la individualidad de los miembros de la familia. Estas situaciones familiares predicen la depresión en los adolescentes. Las interacciones conflictivas familiares tienen una asociación con la depresión (Álvarez, Atienza et al., 2009; Field et al., 2002; Heredia-Ancona et al., 2011; Rosa-Alcázar y Parada-Navas, 2014; Veytia et al., 2012). A su vez, los resultados se confirman con lo expuesto por Field et al. (2002), quienes indican la importancia de los problemas de comunicación entre padres e hijos adolescentes, concluyendo que éstos estaban relacionados con la presencia de síntomas depresivos. El ambiente familiar negativo caracterizado por problemas de comunicación entre padres e hijos es un factor de riesgo para los problemas de salud mental en los hijos, como síntomas de depresión, ansiedad y estrés (Estévez, Musitu y Herrero, 2005).

Los resultados también son consistentes con los hallazgos obtenidos al identificar los sucesos negativos del área familiar como significativos en los adolescentes con depresión (Heredia-Ancona et al., 2011). Al igual que lo ya reportado, la variable "falta de comunicación" en la población adolescente obtuvo el mayor importancia predictiva para la depresión; los jóvenes reportaron que cuanto mayor es la comunicación y la autonomía percibidas por el adolescente, tanto del padre como de la madre, menor es la sintomatología depresiva (Andrade, González et al., 2012).

El análisis del ambiente familiar de acuerdo con el sexo presenta diferencias importantes. Así, las variables que predicen la depresión en los varones se circunscriben a la dimensión afectiva de las relaciones intrafamiliares, como la falta de aceptación familiar, cooperación, alternativas a los problemas familiares y la falta de respeto a la individualidad. También se integra el conflicto entre los padres, el discurso reiterado de los conflictos, los desacuerdos y la falta de afecto en el contexto familiar. Sin embargo estas variables del ambiente familiar tienen escasa importancia predictiva en la depresión, lo cual pudiera indicar que los adolescentes hombres tienen escasa sensibilidad hacia el ambiente familiar negativo, y en general a los acontecimientos negativos, porque la mayoría de los varones se ubica en la escala de depresión sobre el rango debajo de lo normal, con lo cual se considera conveniente ampliar el estudio de la depresión a factores socioculturales, como la percepción social de sí mismo y su relación con los estereotipos, la función del estatus social, las habilidades interpersonales y la influencia social de los pares.

En el caso de las mujeres, los factores del ambiente familiar que predicen la depresión son la falta de normas y reglas de funcionamiento familiar, que indican la ausencia de límites y respeto en la relación interpersonal familiar, la falta de comunicación familiar con los adolescentes en sus actividades cotidianas, así como en las emociones y afectos que presentan, es posible que perciban una escasa disponibilidad a ser escuchadas en un ambiente familiar desorganizado y carente de límites; también se agrega la falta de aceptación familiar con un ambiente sin alternativas de solución a los problemas familiares, carente de cooperación y respeto a la individualidad de los miembros de la familia. Estas variables explican la cuarta parte de la depresión en el caso de las 
mujeres adolescentes, a lo cual se suma el hecho de que las mujeres a temprana edad asumen tareas domésticas y de crianza de los hermanos o familiares; ello ocurre bajo premisas culturales de convivencia definidas en los papeles del ser hombre o mujer, establecidos y trasmitidos de manera generacional en el contexto sociocultural (García-Méndez et al., 2011).

Así, el entorno familiar es de gran trascendencia para la salud de los adolescentes, sobre todo para las mujeres, mientras en los hombres la no aceptación familiar y el conflicto entre los padres se consideran como variables que predicen la depresión con un escaso valor predictivo para la depresión. Al respecto, quienes estudiaron factores asociados con la depresión en hombres identificaron un mínimo soporte familiar asociado con la depresión. Consideran que el grupo familiar no tiene las habilidades para ofrecer apoyo porque la dinámica familiar y el escaso soporte familiar se relacionan con una tendencia a evitar el pedir ayuda para no ir en contra de los preceptos sociales (Londoño y González, 2016).

Los factores sociales y culturales rígidos de la sociedad impiden que los varones expresen la sintomatología depresiva, pues ésta se presenta con síntomas que socialmente son percibidos como expresiones que vulneran la masculinidad. Con estas creencias culturales es posible que la depresión tenga otras dimensiones con manifestaciones distintas, lo que podría llevar a un subregistro de la misma, sobre todo en los varones.

En resumen, se confirma la relevancia de una convivencia familiar afectiva en un ambiente de aceptación, comunicación, respeto, con límites que favorezcan el intercambio y la convivencia entre los miembros. La comunicación se presenta como una variable mediadora de la expresión afectiva y la organización de las relaciones interpersonales.

Es necesario hacer esfuerzos desde diferentes ámbitos públicos y privados para construir una cultura de convivencia familiar saludable, transformando los estilos de relación interpersonal negativos y las condiciones de subordinación de las mujeres en la familia, los cuales vulneran la integridad mental de los adolescentes, llegando a presentar algún tipo de trastorno afectivo a temprana edad.

\section{Referencias}

Álvarez, M., Atienza, G., Heras, E., Fernández, R., Ferrer, E. \& Goicoechea, A. (2009). Guía de práctica clínica sobre la depresión mayor en la infancia y la adolesencia. España: Ministerio de Sanidad y Política Social.

Álvarez, M., Ramírez, B., Silva, A., Cuffin, N. \& Jiménez, M. (2009). La relación entre depresión y conflictos familiares en adolescentes. Journal of Psychology and Psychological Therapy, 9 (2), 205-216.
Andrade, P., Betancourt, D., Vallejo, A., Segura, B. \& Rojas, R. (2012). Prácticas parentales y sintomatología depresiva en adolescentes. Salud Mental, 35 (1), 29-36.

Bella, E.M. (2010). Intento de suicidio en niños y adolescentes: Depresión y trastorno de conducta disocial como patologías más frecuentes. Archivos , G., Argentinos de Pediatría, 108 (2), 124-129.

Benjet, C., Borges, G., Medina-Mora, M.E., Fleiz-Bautista, C. \& Zambrano-Ruiz, J. (2004). La depresión con inicio temprano: Prevalencia, curso natural y latencia para buscar tratamiento. Salud Pública de México, 5, 417-424.

Benjet, C., Borges, G., Medina-Mora, M.E., Méndez, E., Fleiz, C., Rojas, E. \& Cruz, C. (2009). Diferencias de sexo en la prevalencia y severidad de trastornos psiquiátricos en adolescentes de la Ciudad de México. Salud Mental, 32 (2), 155-163.

Borges, G., Benjet, C., Medina-Mora, M.E., Orozco, R. \& Nock, M.K. (2008). Suicide ideation, plan and attempt in the Mexican Adolescent Mental Health Survey. Journal of the American Academy of Child and Adolescent Psychiatry, 47 (1), 41-52.

Bridge, J.A., Goldstein, T.R. \& Brent, D.A. (2006). Adolescent suicide and suicidal behavior. Journal of Child Psychology Psychiatry, 47, 372-394.

Butcher, J., Williams, C., Graham, J., Archer, R., Tellegen, A., BenPorath, Y. \& Kaermmer, B. (1992). Minnesota Multiphasic Personality Inventory-Adolescent: MMPI-A. Mineapolis: Minessota University.

Cárdenas, E., Feria, M., Vázquez, J., Palacios, L. \& De la Peña, F. (2010). Guía clínica para los trastornos afectivos (depresión y distimia) en niños y adolescentes. México: Instituto Nacional de Psiquiatría Ramón de la Fuente Muñiz.

Cohen, R. \& Swerdlik, M. (2006). Pruebas y evaluación psicológicas. Introducción a las pruebas y la medición. México: McGraw Hill Interamericana.

Essau, C., Conradt, J. \& Petermann, F. (2000). Frequency, comorbidity, and psychosocial impairment of depressive disorders in adolescents. Journal of Adolescent Research, 15 (4), 470-481.

Estévez, E., Musitu, G. \& Herrero, J. (2005). El rol de la comunicación familiar y del ajuste escolar en la salud mental del adolescente. Salud Mental, 28 (4), 81-89.

Field, T., Diego, M. \& Sanders, C. (2002). Adolescent's parent peer relationships. Adolescence, 37 (145), 121-130.

Galicia, I., Sánchez, A. \& Robles, F. (2009). Factores asociados a la depresión en adolescentes: Rendimiento escolar y dinámica familiar. Anales de Psicología, 25 (2), 227-240.

Galicia, I., Sánchez, A. \& Robles, F. (2013). Autoeficacia en escolares adolescentes: Su relación con la depresión, el rendimiento académico y las relaciones familiares. Anales de Psicología, 29 (2), 491-500.

García-Méndez, M., Rivera-Aragón, S., Reyes-Lagunes, I. \& DíazLoving, R. (2011). El enfrentamiento y el conflicto: Factores que intervienen en la depresión. Acta de Investigación Psicológica, 1 (3), 415-427.

Garrison, Z.F., Walter, J.Z., Cuffe, S.P., MacKnown, R.E., Addy, C.L. \& Jackson, K.L. (1997). Incidence of depressive disorders and dysthimia in young adolescents. American Academy 
of Child and Adolescent Psychiatry, 36 (4), 458-465.

González-Forteza, C., Jiménez-Tapia, J.A., Ramos-Lira, L. \& Wagner, F.A. (2008). Aplicación de la escala de depresión del Centro de Estudios Epidemiológicos en Adolescentes de la Ciudad de México. Salud Pública de México, 50 (4), 292-299.

González-González, A., Juárez, F., Solís, C., González-Forteza, C., Jiménez, A., Medina-Mora, M.E. \& Fernández-Varela, H. (2012). Depresión y consumo de alcohol y tabaco en estudiantes de bachillerato y licenciatura. Salud Mental, 35 (1), 51-55.

Heredia-Ancona, M., Lucio-Gómez, E. \& Suárez, L.E. (2011). Depresión y sucesos de vida estresantes en adolescentes. Revista Latinoamericana de Medicina Conductual, 1 (2), 49-57.

Hopcroff, R.L. \& Bradley, D.B. (2007). The sex difference in depression across 29 countries. Social Forces, 85 (4), 14831507.

Londoño, C., \& González, M. (2016). Prevalencia de depresión y factores asociados en hombres. Acta Colombiana de Psicología, 19 (2), 315-329.

Lucio, E., Ampudia, A. \& Durán, C. (2004). Manual para la administración y calificación del Inventario Multifásico de Minnesota Adolescentes (MMPI-A) Versión en español. México: Manual Moderno.

Marmorstein, N. \& Iacono, W. (2003). Major depression and conduct disorder in a twin sample: Gender, functioning and risk for future psychopathology. Journal of the American Academy of Child and Adolescent Psychiatry, 42 (2), 225-233.

Moreno, A. \& Medina-Mora, M.E. (2008). Tabaquismo y depresión. Salud Mental, 31 (5), 409-415.
Organización Mundial de la Salud (2016). La depresión. Nota descriptiva No. 369. Recuperado de: who.int/mediacentre/ factsheets/fs369/es/

Organización Panamericana de la Salud (2009). Epidemiología de los trastornos mentales en América Latina y el Caribe. Publicación Científica y Técnica No. 632. Washington: OPS.

Pintado-Machado, Y., Jiménez, Y., Padilla, M., Guerra, J. \& Antelo, M. (2010). Trastornos psicológicos en los adolescentes. Una visión general. Salud Mental, 8 (37), 89-100.

Rodríguez, A. \& Granada, O. (2007). Trastornos depresivos en la infancia y la adolescencia. Revista Clínica de Medicina de Familia, 1 (6), 270-276.

Rosa-Alcázar, A. \& Parada-Navas, J. (2014). Síntomas psicopatológicos en adolescentes españoles: Relación con los estilos parentales percibidos y la autoestima. Anuario de Psicología, 30 (1), 133-142.

Ruiz-Cárdenas, C.T., Reidl, L.M. \& Gallegos, R. (2017). Validez de constructo de la escala familiar para adolescentes. Vertientes, 20 (1), 35-42.

Schleicher, H., Harris, K., Catley, D. \& Nazir, N. (2009). The role of depression and negative affect regulation expectancies in tobacco smoking among college students. Journal of American College Health, 57 (5), 507-512.

Veytia, M., González, N., Andrade, P. \& Oudhof, H. (2012). Depresión en adolescentes El papel de sucesos vitales estresantes. Salud Mental, 35 (1), 37-43.

Zarragoitía, I. (2013). Las diferencias de sexo y género en la depresión proclividad en la mujer. Medwave, 13 (3): doi:10.5867/medwave.2013.03.5651 


\section{Meta-Análisis del Artículo}




\section{Dimensión Cuantitativa}

\section{Perfil de Evaluación entre pares}
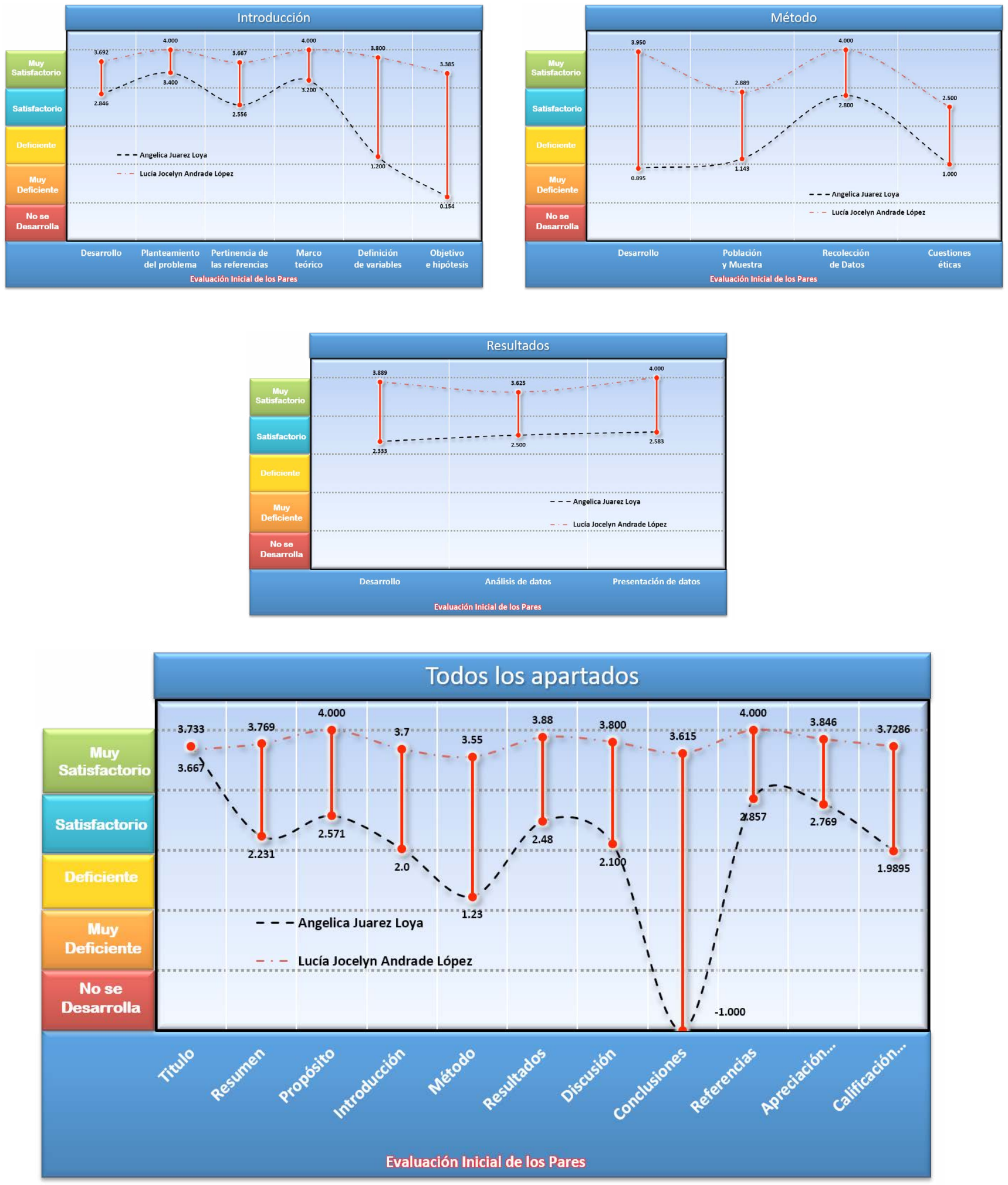


\section{Índice de Concordancia}

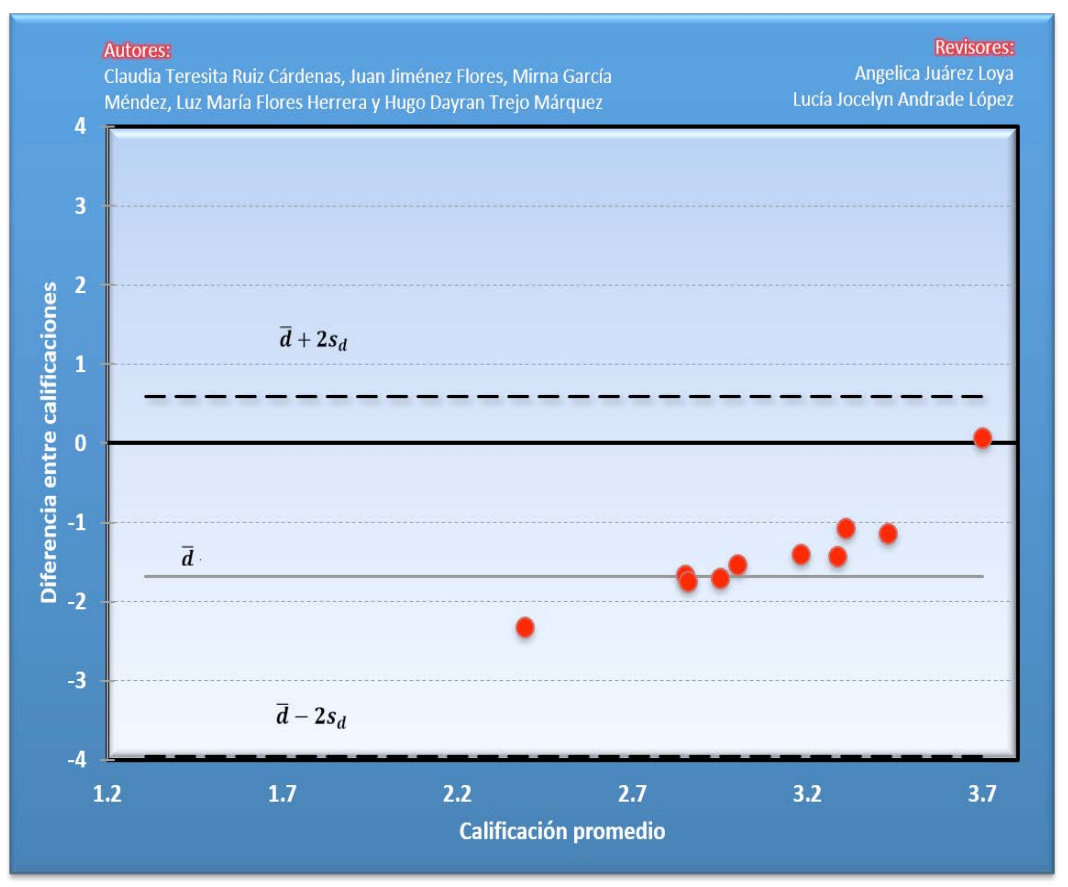

Índice de Acuerdo

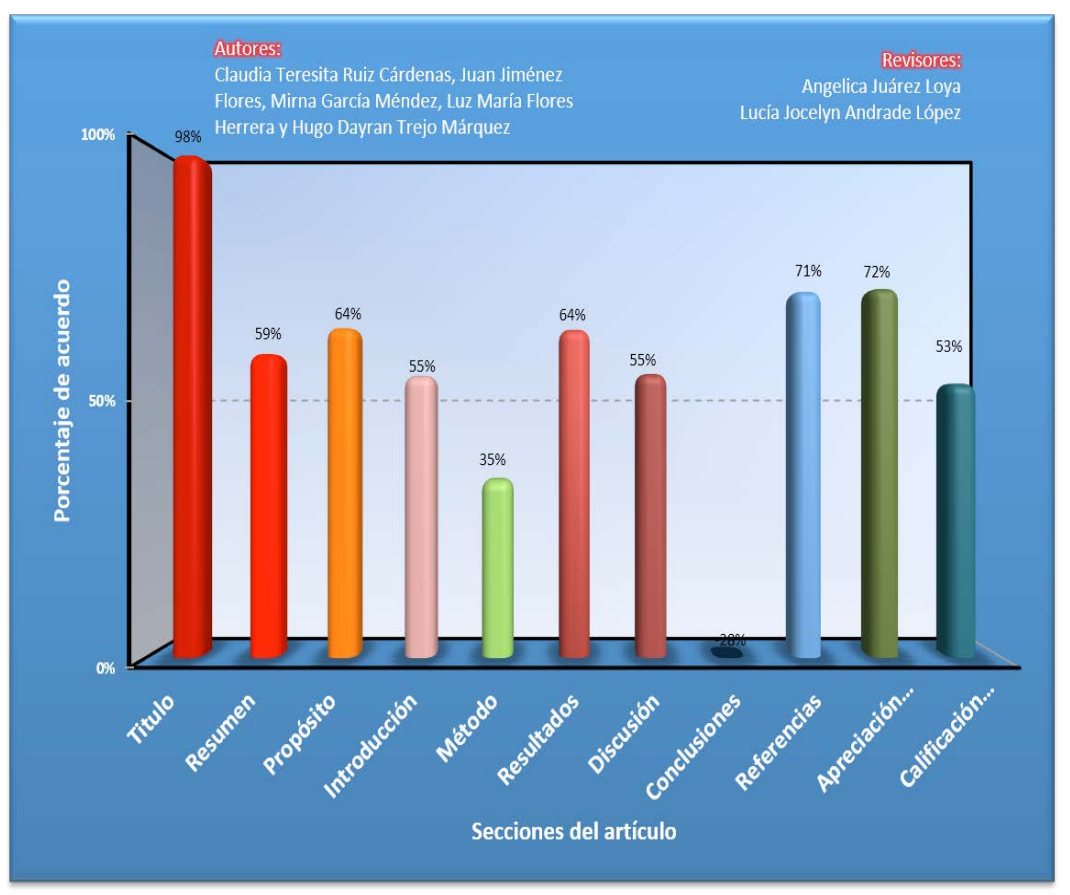




\begin{tabular}{|c|c|}
\hline Revisor 1 & Revisor 2 \\
\hline Angélica Juárez Loya & Lucía Jocelyn Andrade López \\
\hline \multicolumn{2}{|c|}{ Título/Autoría } \\
\hline $\begin{array}{l}\text { El título está bien, sólo en uno de los nombres pusieron } \\
\text { abreviado un nombre, por ello disminuyó el puntaje. }\end{array}$ & $\begin{array}{l}\text { Considero que el título es correcto para hacer referencia al } \\
\text { contenido del artículo. }\end{array}$ \\
\hline \multicolumn{2}{|c|}{ Resumen } \\
\hline $\begin{array}{l}\text { En el resumen en español sugiero que revisen la redac- } \\
\text { ción del tercer párrafo. Debido a que el resumen es la } \\
\text { primera impresión del trabajo, al lector debe quedarle } \\
\text { clara la comparación que hicieron de las condiciones } \\
\text { en el perfil autonómico desde antes. Si no lo dijeron, } \\
\text { entonces este resultado desubica un poco y se hace } \\
\text { complicado comprenderlo. Sugiero no utilizar los valo- } \\
\text { res de r en el resumen y redondear la edad. }\end{array}$ & $\begin{array}{l}\text { De manera general, el resumen cuenta con los elemen- } \\
\text { tos necesarios que han de tomarse en cuenta para esta } \\
\text { sección, sin embargo el contenido del mismo es muy } \\
\text { extenso sobrepasando el límite de palabras, por lo que } \\
\text { se recomienda que sea más resumido y concreto. }\end{array}$ \\
\hline \multicolumn{2}{|c|}{ Próposito del Estudio } \\
\hline $\begin{array}{l}\text { La principal dificultad que observo en el objetivo es que } \\
\text { solo dice que se haría la evaluación de las asociaciones en- } \\
\text { tre variables, sin embargo en el resto del artículo (resultados } \\
\text { y conclusiones), se presentan muchos otros análisis que no } \\
\text { coinciden con el objetivo planteado. Sugiero que si van a re- } \\
\text { portar los análisis descritos, entonces lo enuncien en objeti- } \\
\text { vos específicos. Tampoco aparece pregunta de investigación } \\
\text { (que permita identificar las hipótesis de forma más clara) y a } \\
\text { la hora de reportar los resultados, no muestran el resultado } \\
\text { principal al inicio (que tendría que ser el de la correlación } \\
\text { entre variables), tampoco aparece en la discusión el resulta- } \\
\text { do principal al inicio, para darle la importancia que merece } \\
\text { a esta pregunta de investigación que se hicieron. Sugiero que } \\
\text { los autores evalúen también qué análisis merece la pena re- } \\
\text { portar en su artículo y cuales pueden omitirse pues ocupan } \\
\text { gran parte de la explicación sin ser los objetivos primordia- } \\
\text { les, por ejemplo, las comparaciones por sexo quizá no son } \\
\text { necesarias porque tienen grupos no equiparables para la } \\
\text { comparación (por la cantidad de hombres que participaron } \\
\text { en contraste con las mujeres), también en esta comparación } \\
\text { es difícil que se observen diferencias por los niveles bajos de } \\
\text { depresión que la población reportó, generalmente las dife- } \\
\text { rencias solo se muestran cuando sí hay personas con depre- } \\
\text { sión (igualmente con ansiedad). }\end{array}$ & \begin{tabular}{|l} 
|l \\
Esta sección cumple con puntos importantes que per- \\
miten entender el propósito de la investigación, su sur- \\
gimiento y la necesidad de realizarla. \\
\\
gimal
\end{tabular} \\
\hline
\end{tabular}




\begin{tabular}{|c|c|}
\hline Revisor 1 & Revisor 2 \\
\hline \multicolumn{2}{|c|}{ Introducción } \\
\hline $\begin{array}{l}\text { En el marco teórico, sugiero que revisen el orden de las } \\
\text { ideas de las más generales a las ideas particulares y tam- } \\
\text { bién que consideren incluir solo la información que deja } \\
\text { ver al lector la importancia del estudio, incluso resaltando } \\
\text { más una riqueza importante que tienen en sus medicio- } \\
\text { nes: hicieron medición fisiológica (que no todos los estu- } \\
\text { dios tienen la oportunidad de llevar a cabo). Aunque las } \\
\text { referencias me parecen apropiadas y actuales, es necesario } \\
\text { tratar de llevar el relato de los antecedentes con un poquito } \\
\text { más de ilación. No tienen descritas las hipótesis. }\end{array}$ & $\begin{array}{l}\text { Considero que habría que ser más claros y precisos en } \\
\text { cuanto a la formulación de las hipótesis de investigación } \\
\text { ya que en el texto no se pueden conocer como parte de la } \\
\text { investigación que se llevó a cabo. }\end{array}$ \\
\hline \multicolumn{2}{|c|}{ Método } \\
\hline $\begin{array}{l}\text { Sugiero que describan las propiedades psicométricas } \\
\text { de las escalas que utilizaron, que no repitan materiales } \\
\text { y mediciones y que amplíen la información de aspectos } \\
\text { éticos atendiendo a los preceptos de la Declaración de } \\
\text { Helsinky y el Código de Nurenberg. También me pa- } \\
\text { rece que describen con extremo detalle aspectos del } \\
\text { procedimiento de la investigación (como lo del per- } \\
\text { miso a los profesores y afectación de sus clases) que } \\
\text { no aportan mucho a la información que necesitamos } \\
\text { saber como lectores, pero les falta la parte de protec- } \\
\text { ción de la información de los estudiantes, por poner } \\
\text { un ejemplo. Vuelvo al punto de los análisis estadísticos, } \\
\text { describieron muchos análisis que no están justificados } \\
\text { en el planteamiento de su investigación y en el objetivo } \\
\text { que describen, sería importante que todo coincidiera } \\
\text { desde el planteamiento hasta los resultados. Respecto } \\
\text { a la descripción de los grupos de participantes por pe- } \\
\text { riodo escolar, en una parte del artículo dicen que son } \\
\text { periodos anuales y más adelante hablan de semestres, } \\
\text { luego hablan de que no pudieron tomar datos de los } \\
\text { alumnos de últimos semestres que salen a prácticas o } \\
\text { clases fuera de la universidad (pero que probablemente } \\
\text { sean ellos los que enfrentan más estresores que pueden } \\
\text { generar los malestares en salud) y no justifican por qué } \\
\text { (no es una justificación suficientemente válida para el } \\
\text { diseño, que no están en la universidad). }\end{array}$ & $\begin{array}{l}\text { En términos generales, el método ha sido explicado de } \\
\text { manera clara permitiendo conocer cómo fue el proce- } \\
\text { dimiento que se siguió en la investigación. } \\
\end{array}$ \\
\hline
\end{tabular}




\begin{tabular}{|c|c|}
\hline Revisor 1 & Revisor 2 \\
\hline \multicolumn{2}{|c|}{ Resultados } \\
\hline $\begin{array}{l}\text { Algunas de las tablas que presentan podrían evitarse, por } \\
\text { ejemplo la del protocolo de reclutamiento de participan- } \\
\text { tes. Mi evaluación con respecto a los análisis estadísticos } \\
\text { es porque me parece que no justificaron por qué hacer to- } \\
\text { dos esos análisis desde su objetivo. Y como comenté pre- } \\
\text { viamente, en ocasiones no están considerando el tamaño } \\
\text { de sus grupos para elegir sus análisis, por ejemplo en la } \\
\text { comparación por sexos. Respecto a las tablas, si hacen } \\
\text { correlaciones es apropiado tener la tabla de correlación, } \\
\text { si hacen ANOVA, es mejor hacer la tabla ANOVA como } \\
\text { sugiere APA reportar ese resultado, tienen algunas tablas } \\
\text { que podrían evitarse para incluir estas del análisis. }\end{array}$ & Sin comentarios. \\
\hline \multicolumn{2}{|c|}{ Discusión } \\
\hline $\begin{array}{l}\text { En la discusión, sugiero que comiencen explicando si se } \\
\text { afirman o niegan las hipótesis que tenían, también es im- } \\
\text { portante que traten de no especular sobre los trastornos } \\
\text { de ansiedad y depresión, porque no es eso lo que evalua- } \\
\text { ron con sus mediciones y porque los datos que recopila- } \\
\text { ron hablan solamente de niveles moderados de ansiedad y } \\
\text { casi nulos de depresión. Me parece que en la discusión de- } \\
\text { bieran evitar hablar de los sexos diferenciadamente (por } \\
\text { las razones que les expuse previamente), que traten de dar } \\
\text { explicación a por qué los jóvenes de esa universidad no } \\
\text { reportan niveles elevados de ansiedad y depresión, si ge- } \\
\text { neralmente los estudios hablan de ese tipo de problemas, } \\
\text { también será importante que eviten hablar de procesos } \\
\text { de intervención (ej. yoga y terapia cognitivo-conductual) } \\
\text { pues su tesis no perseguía precisamente ese objetivo y apa- } \\
\text { rece descontextualizado de lo que están diciendo. Falta } \\
\text { describir las limitaciones del estudio. }\end{array}$ & $\begin{array}{l}\text { La discusión sitúa en una reflexión argumentada acerca } \\
\text { de los objetivos del artículo ya que se muestra de manera } \\
\text { estructurada y clara los hallazgos de la investigación para } \\
\text { posteriormente ser comparados con los hallazgos de otras } \\
\text { investigaciones que ya han sido realizadas logrando que se } \\
\text { identifiquen de manera sencilla para el lector. }\end{array}$ \\
\hline
\end{tabular}




\begin{tabular}{|c|c|}
\hline Revisor 1 & Revisor 2 \\
\hline \multicolumn{2}{|c|}{ Conclusiones } \\
\hline $\begin{array}{l}\text { Sugiero que revisen los criterios que se evalúan en esta } \\
\text { sección para desarrollarla con base en estos y que eso } \\
\text { ayude a completar esta sección. }\end{array}$ & Sin comentarios. \\
\hline \multicolumn{2}{|c|}{ Referencias } \\
\hline $\begin{array}{l}\text { En algunas referencias faltó un punto o coma y también } \\
\text { sugiero revisar el manual APA para cuando una refe- } \\
\text { rencia tiene más de } 5 \text { autores. Las siguientes referencias } \\
\text { no aparecen en el texto o tienen un error en el apellido, } \\
\text { revisar: Regehr, C., Glancy, D. \& Pitts, A. (2013) Inter- } \\
\text { ventions to reduce stress in university students: A re- } \\
\text { view and meta-analysis. Journal of Affective Disorders, } \\
\text { 148(1): 1-11. doi: } 10.1016 / \text { j.jad.2012.11.026. Sevinc, } \\
\text { G., Hölzel, B. K., Hashmi, J., Greenberg, J., McCallis- } \\
\text { ter, A., Treadway, ... Lazar, S. W. (2018). Common and } \\
\text { dissociable neural activity after mindfulness-based } \\
\text { stress reduction and relaxation response programs. } \\
\text { Psychosomatic medicine, } 80(5), 439-451 \text {. doi: } 10.1097 / \\
\text { PSY.0000000000000590 }\end{array}$ & Sin comentarios. \\
\hline
\end{tabular}




\section{Historia del Proceso}

\section{EDITORIAL}

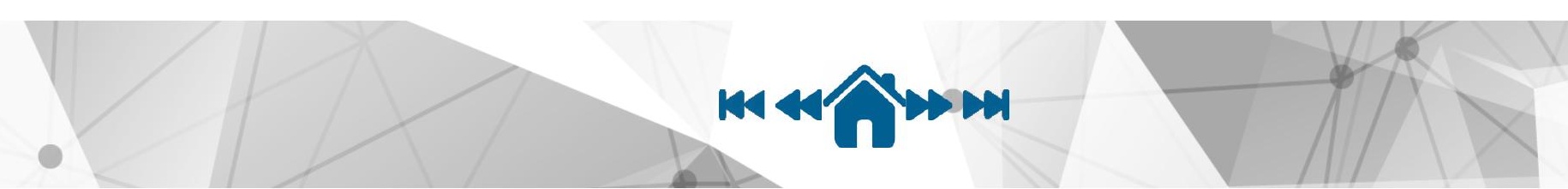

